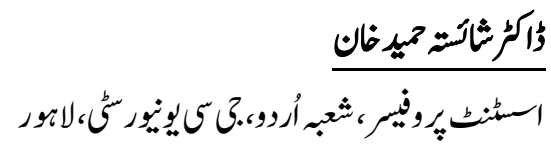

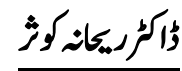

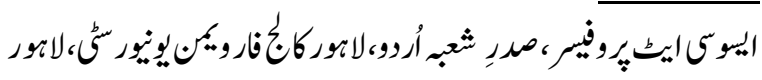

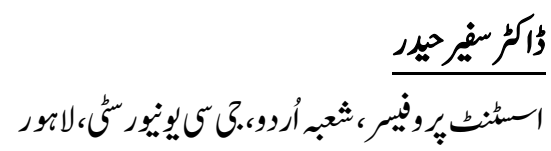

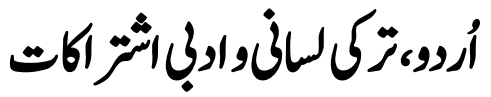

Dr. Shaista Hameed Khan

Assistant Professor, GC University, Lahore.

Dr. Rehana Kausar

Associate Professor, Lahore Collage for women University, Lahore.

Dr. Safeer Haider

Assistant Professor, GC University, Lahore.

\title{
Urdu-Turkish linguistic and literary collaborations
}

People who speak and understand Urdu language live in every part of the world. Beside this, Urdu language teaching is continuing in different countries of the world. There is a common tendency for reading, writing and learning Urdu in Turkey and the other countries of the world. Turkish universities have proper departments of Urdu. Linguistically, many commonalities are found in Urdu and Turkish language which has several reasons. In this article the common linguistic properties in Urdu and Turkish language have been proven according to syntax. Apart from this, this article also has a brief discussion about the literary, civilization and central relationship in the languages of these two countries.

Keywords: Vrdu, Turkey, Turkish, Language, Linguistic, Literature, Literary, Collaboration, civilization.

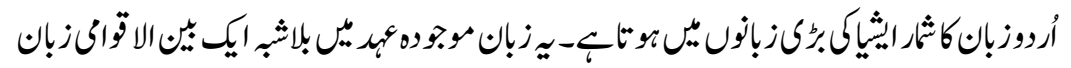

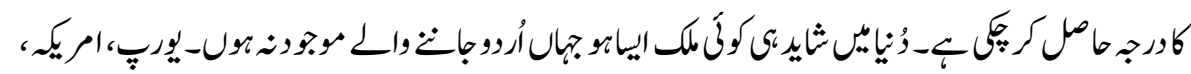




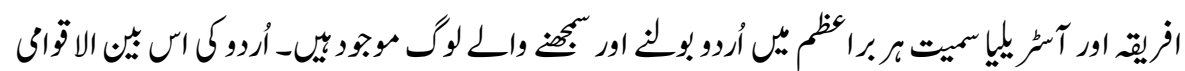

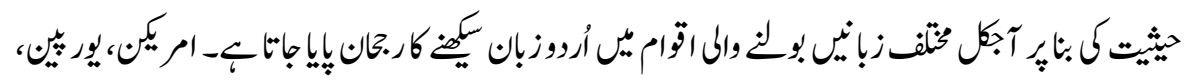

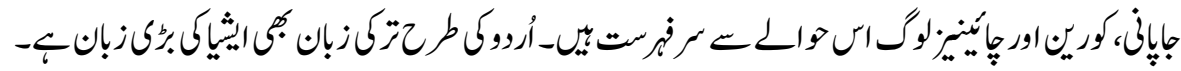

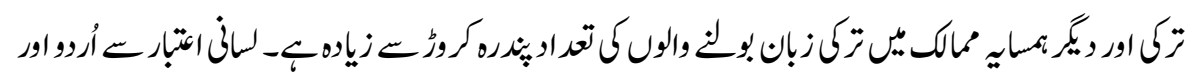

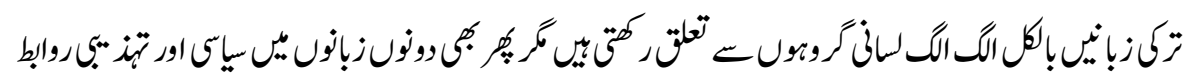

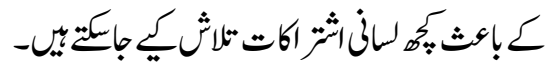

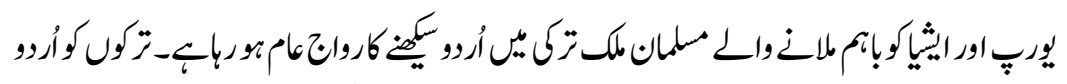

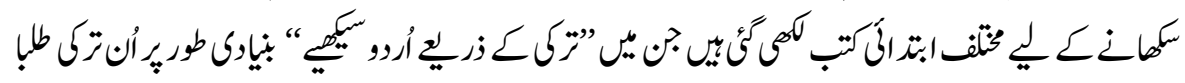

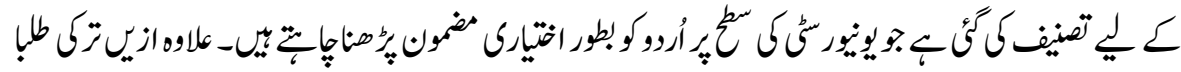

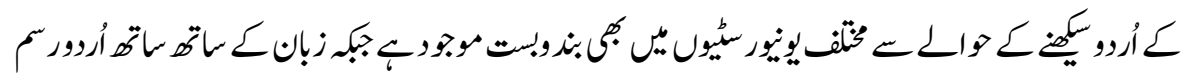

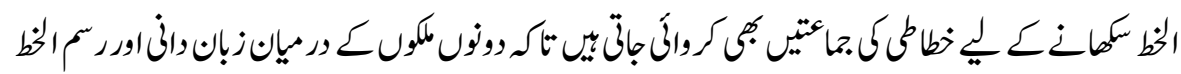

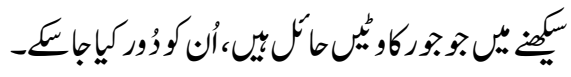

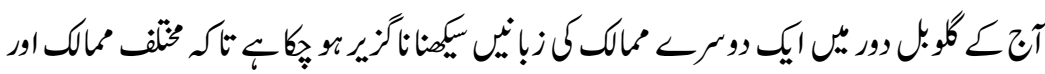

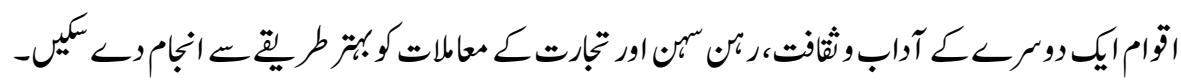

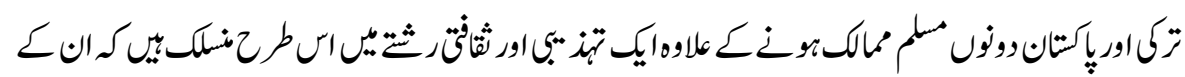

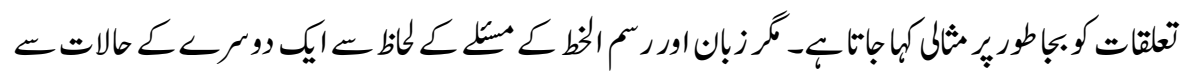

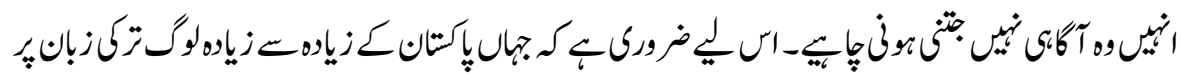

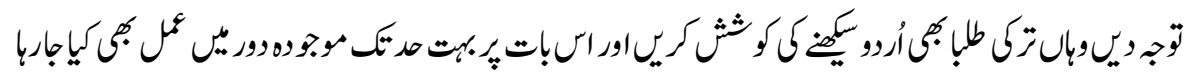

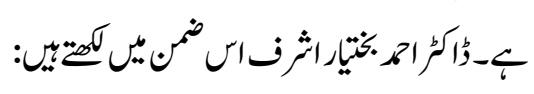

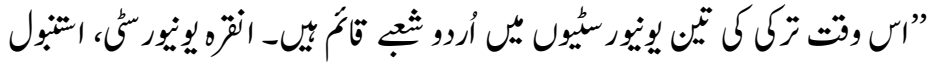

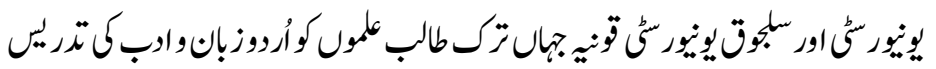

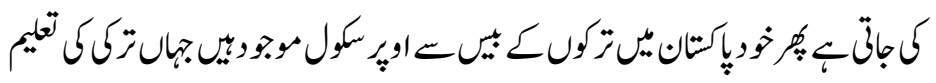

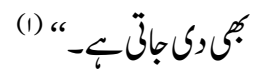




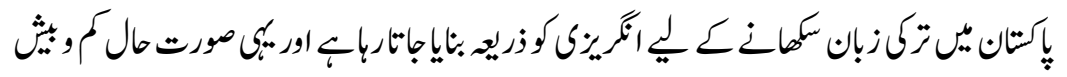

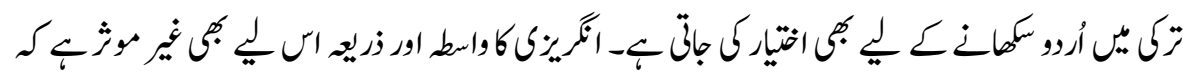

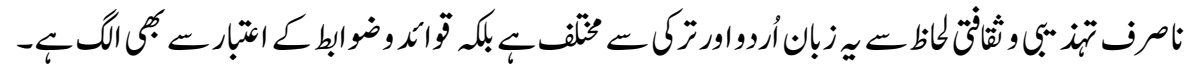

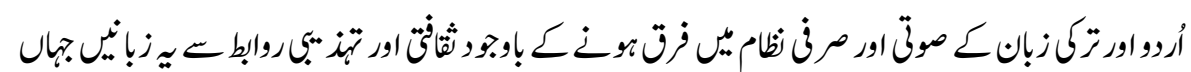

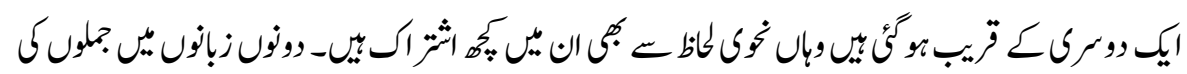

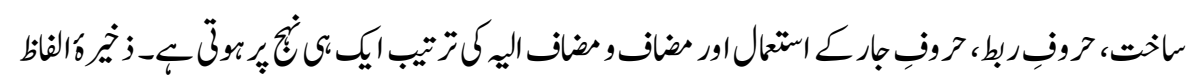

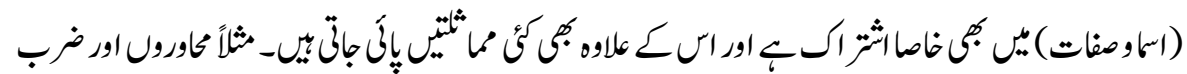

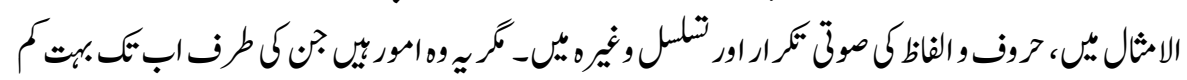

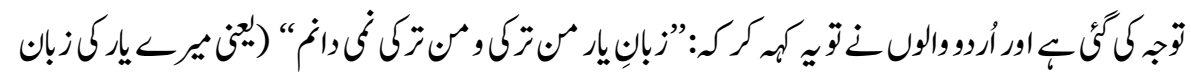

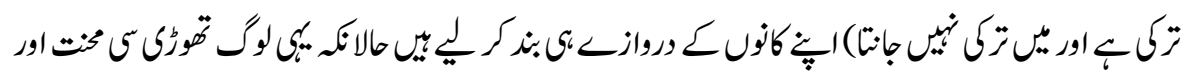

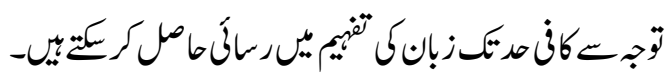

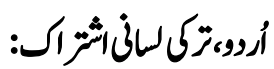

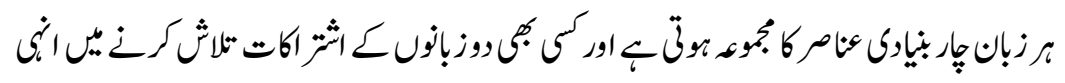

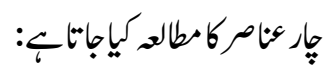

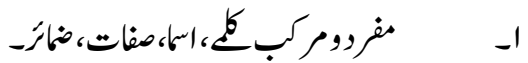

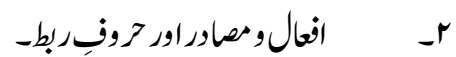

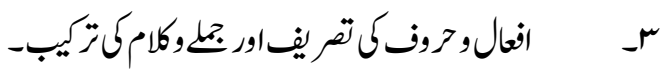

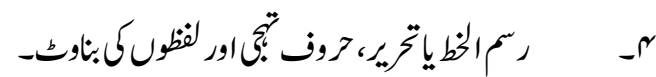

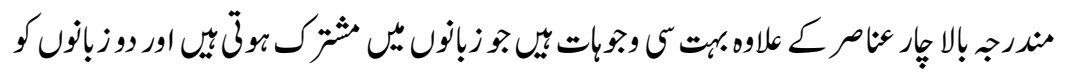

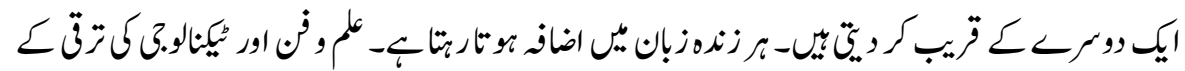

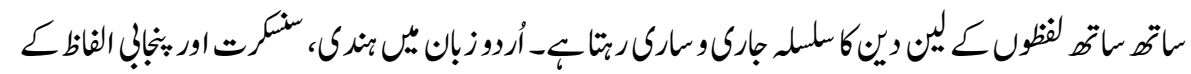

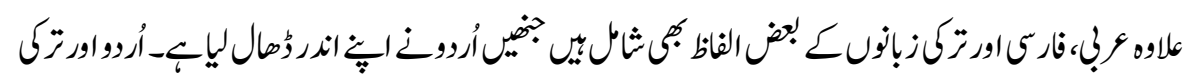

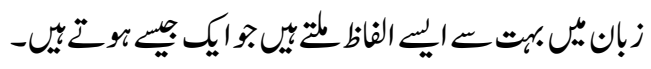




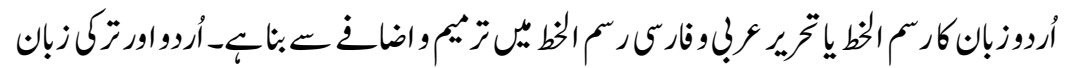

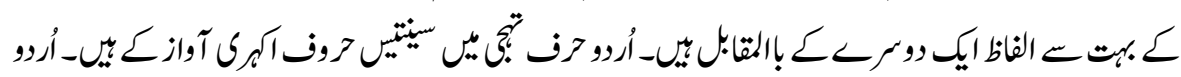

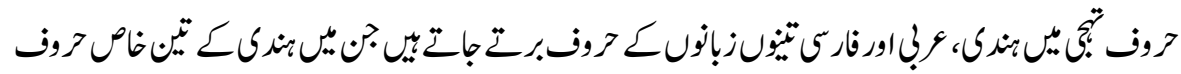

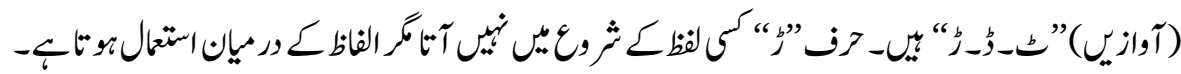

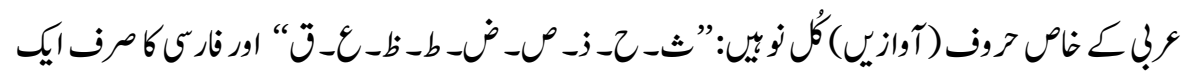

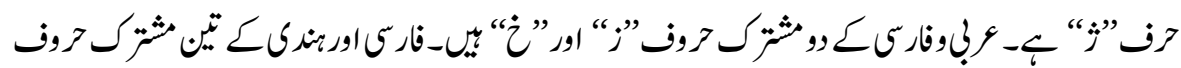

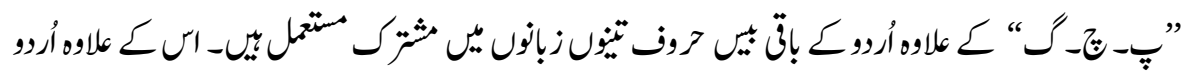

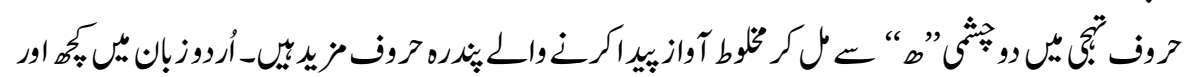

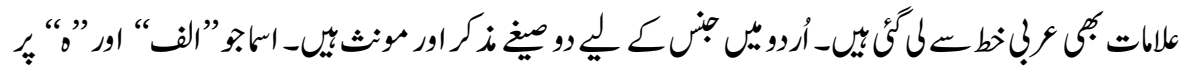

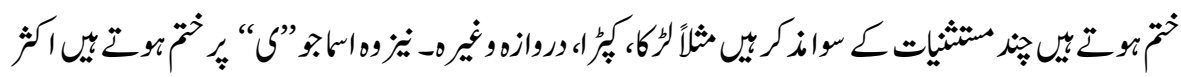

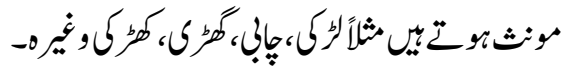
اسمذك: OGUL 国回OGUL 6 ADAM آرى كورثا

الممونث:

\author{
KIZ
}

بئي

KIZ

(5)

KADIN

ورت

KISRAK

كورئ

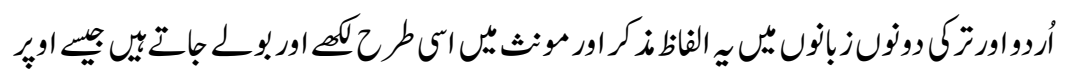

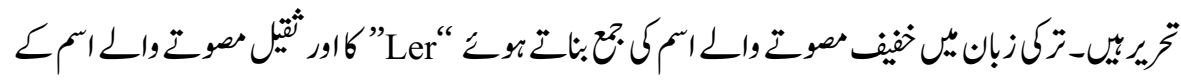

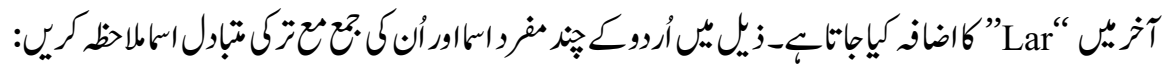

EVLER

$$
\text { كمرب+ع }
$$

SEHIRLER

EV

الم مغر

$$
\text { ثمبر }
$$

SEHÌR 


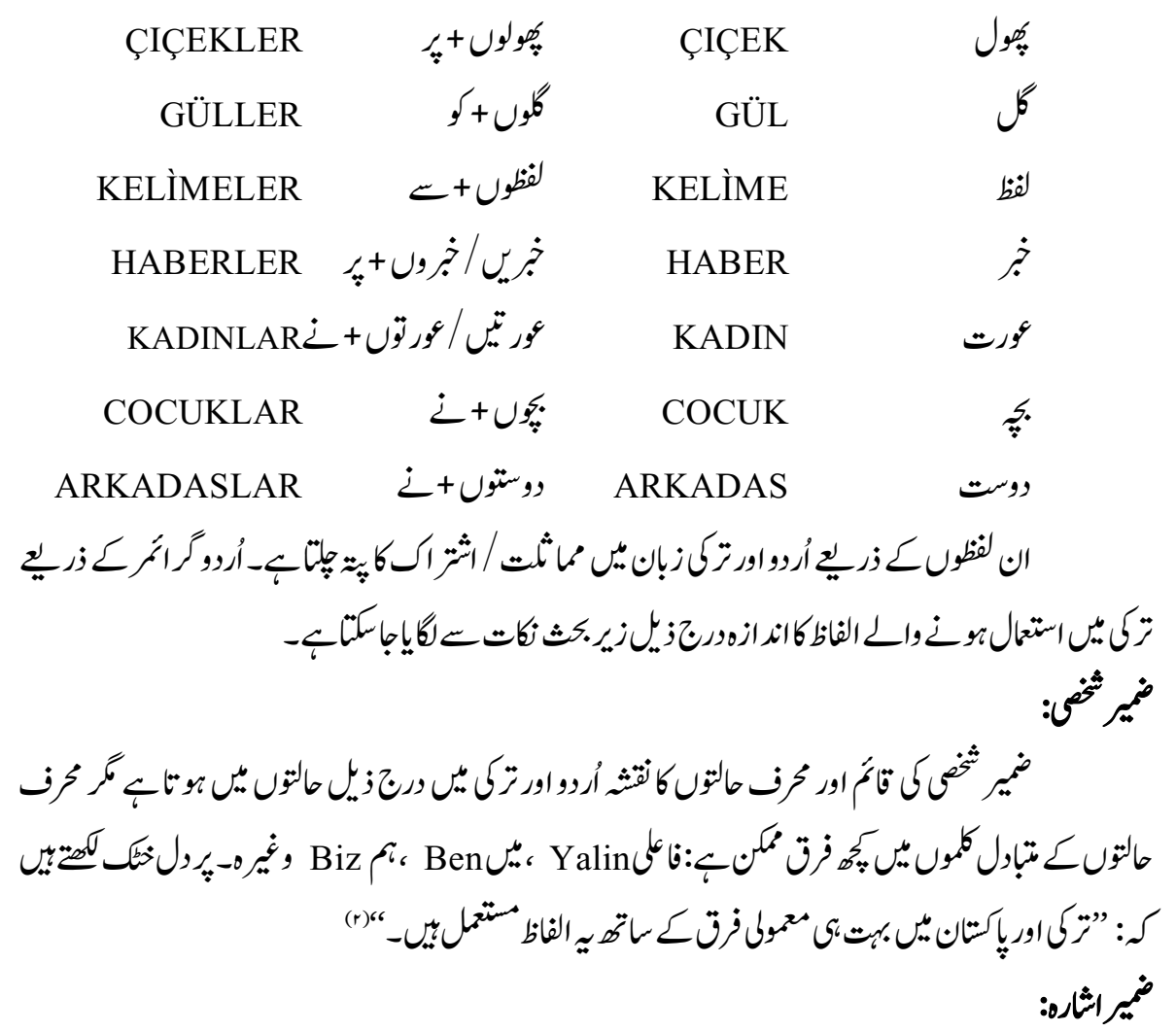

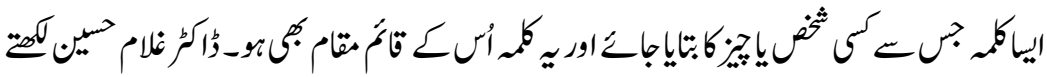

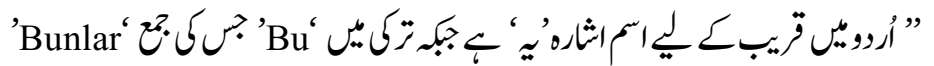

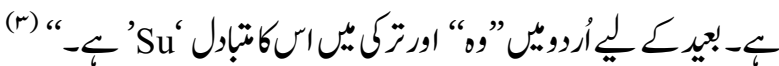

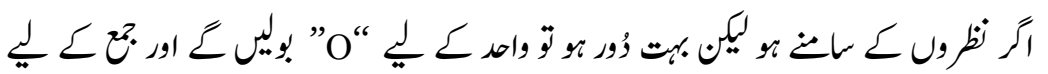

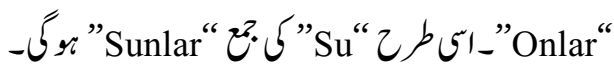




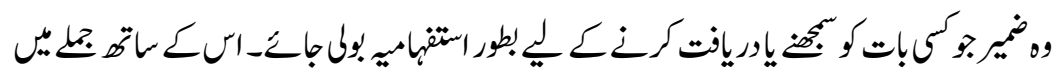

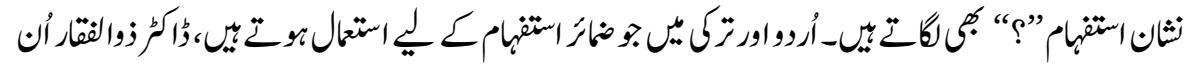
كمابت كميتبي:

\begin{tabular}{|c|c|c|}
\hline KIME? & كِع & KIM? \\
\hline HANGI? & كون سا؟ & KIMIN? \\
\hline $\mathrm{KAC}$ ? & كت:ا؟ & NE? \\
\hline NERE?" & كمان؟ & NASIL? \\
\hline
\end{tabular}

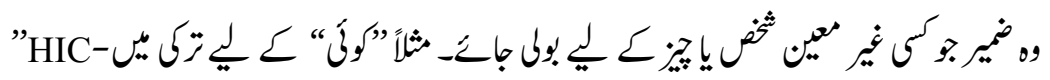

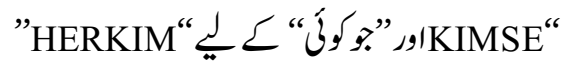
مير استزاقّ:

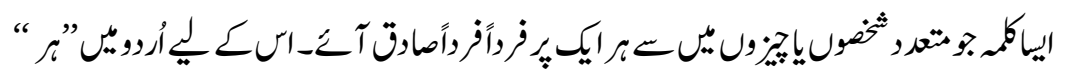

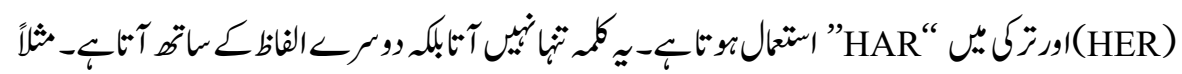

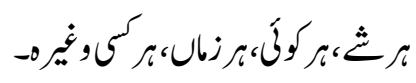

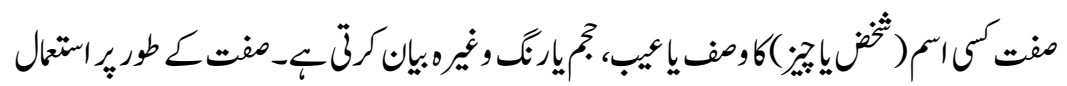

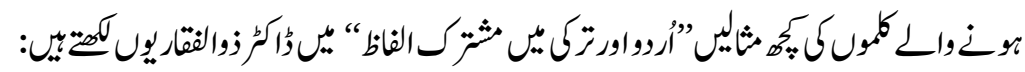

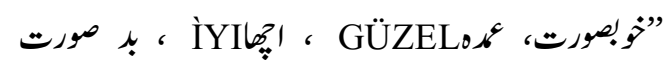

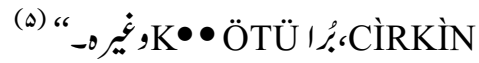

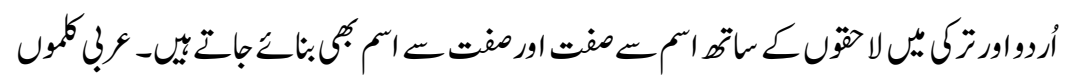

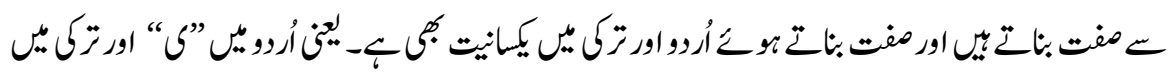

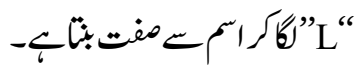




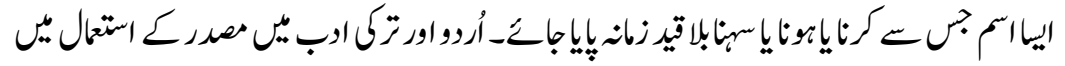

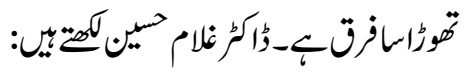

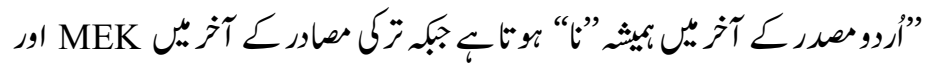

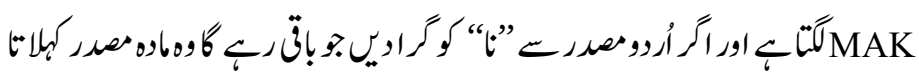

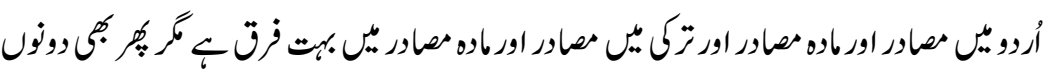

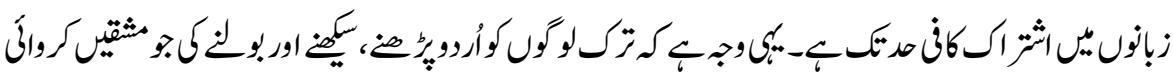

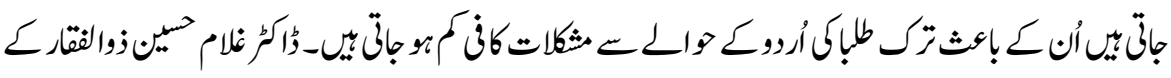

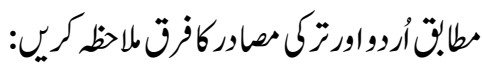

\begin{tabular}{|c|c|}
\hline SINAMAK_SINA & "آزانا... آزا" \\
\hline İNMEK_ İ̀ & أترنا... أُت \\
\hline YIKMAK_YIK & أجارُنا.... أجارُ \\
\hline UÇMAK_UÇ & 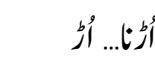 \\
\hline YAGMAK YAG“ & \\
\hline
\end{tabular}

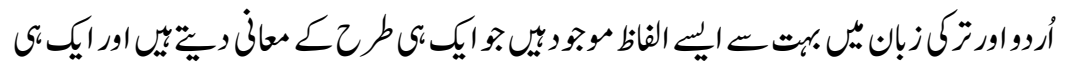

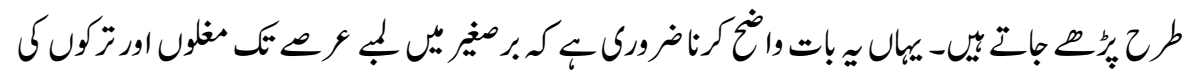

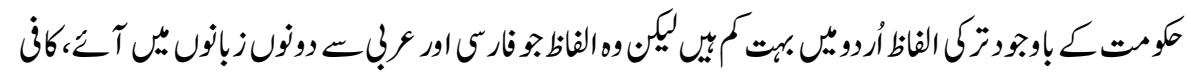

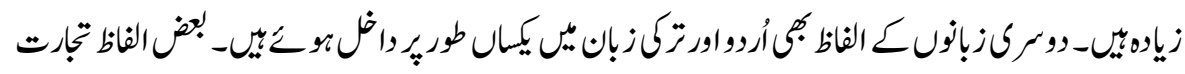

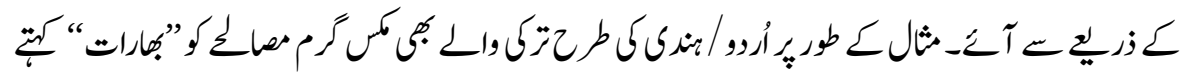

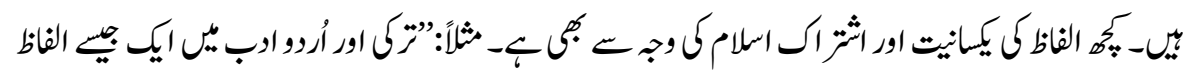

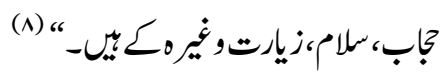




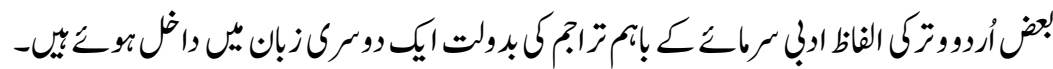

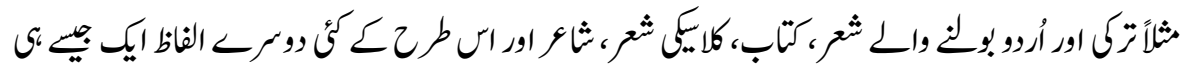

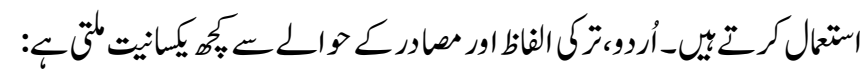

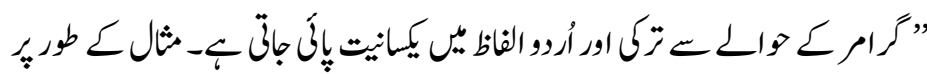

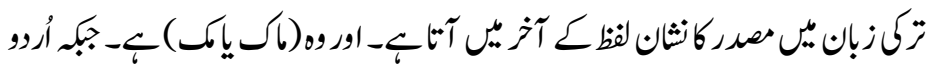

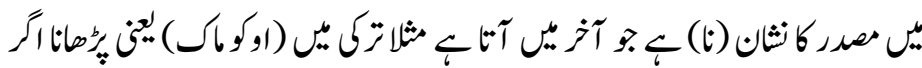
(و)

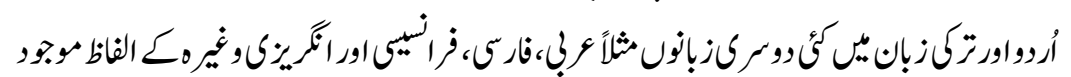

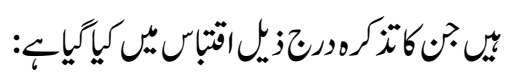

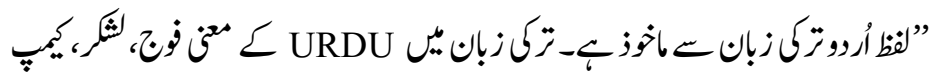

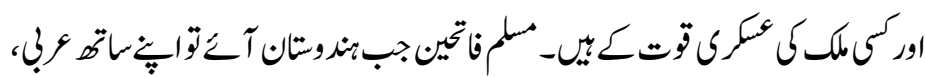

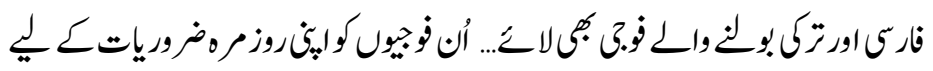

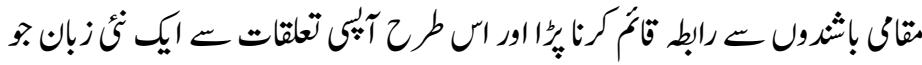

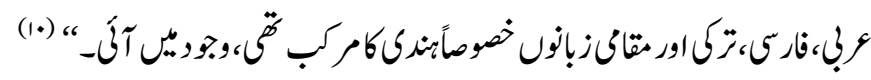

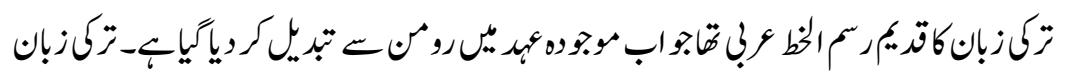

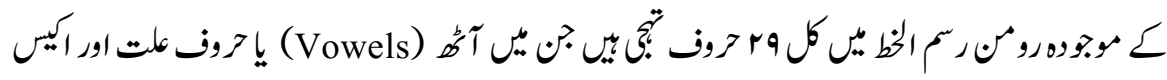

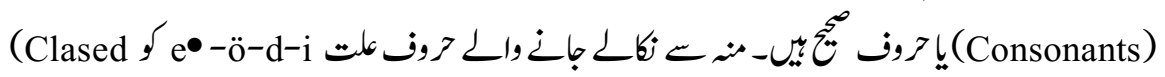
Vowels) (Front Vowels)

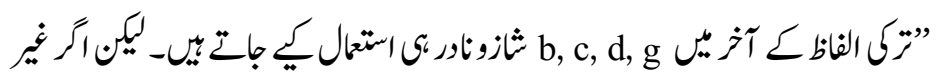

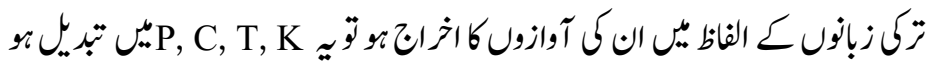

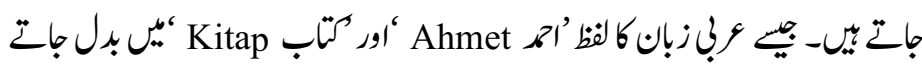
(11) ، ب. 


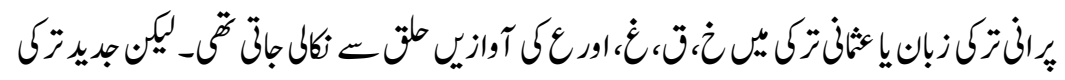

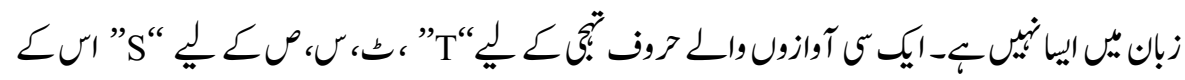

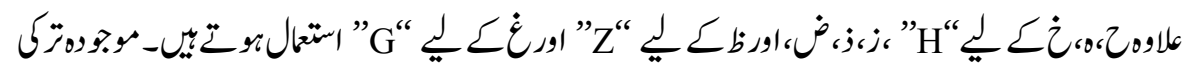

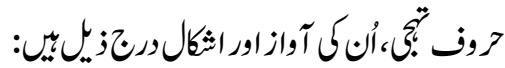

براحف

$\mathrm{A}$

جموناجف

آواز

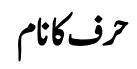

íl

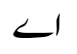

B

b

$\div$

$\mathrm{C}$

c

ب

$\leftarrow$

C

D

c

d

$?$

E

e

<象

F

G

$\mathrm{f}$

$<1$

إ

$\mathrm{f}$

ف

i

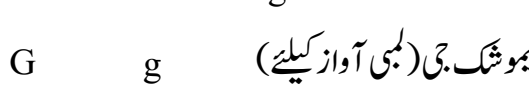

3.

$\mathrm{H}$

I

I

J

K

L

M

$\mathrm{N}$

$\mathrm{O}$

$\mathrm{h}$
$\mathrm{I}$
$\mathrm{I}$
$\mathrm{j}$
$\mathrm{k}$
$\mathrm{1}$
$\mathrm{m}$
$\mathrm{n}$
$\mathrm{o}$

$\mathrm{h}$

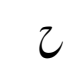

ใ.

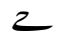

$i$

I

J

K

1

1

n

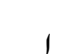

I

j

L;

;

c.6

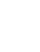

$\checkmark$

$L$

m

(1)

o

J

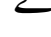

$<$

i

$1 \%$ 


\begin{tabular}{|c|c|c|c|}
\hline Ö & $\ddot{o}$ & أو & الماوتتاو \\
\hline $\mathrm{P}$ & $\mathrm{p}$ & 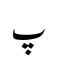 & 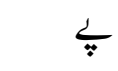 \\
\hline $\mathrm{R}$ & $\mathrm{r}$ & ر & $<$ \\
\hline $\mathrm{S}$ & $\mathrm{s}$ & v & - \\
\hline $\mathrm{S}$ & $\mathrm{s}$ & $\dot{\xi}$ & ث \\
\hline $\mathrm{T}$ & $\mathrm{t}$ & b & $\mathcal{L}$ \\
\hline Ü & $\ddot{\mathrm{u}}$ & ليو & يو \\
\hline $\mathrm{U}$ & $\mathrm{u}$ & اُو & الطاوَتِيو \\
\hline V & $\mathrm{v}$ & , & وك \\
\hline Y & $\mathrm{y}$ & اك & $\ddot{\jmath}$ \\
\hline Z & $\mathrm{z}$ & j & زك \\
\hline
\end{tabular}

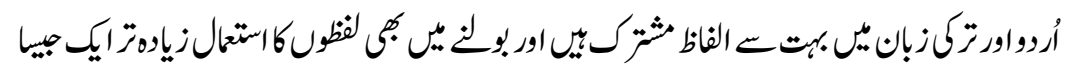

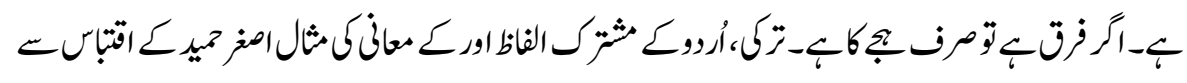
6لاحظ كرئ:

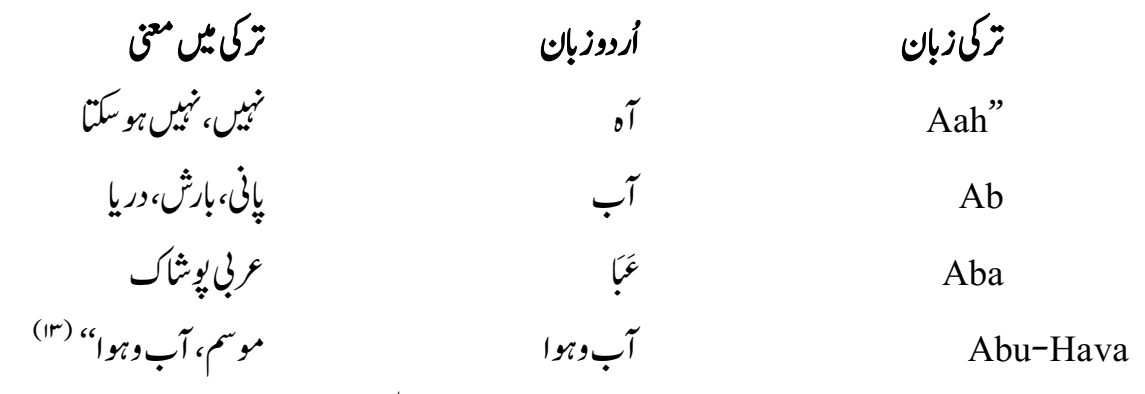

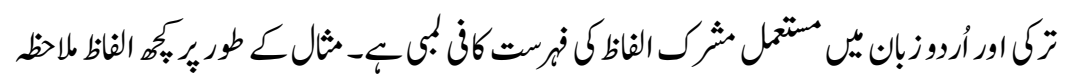




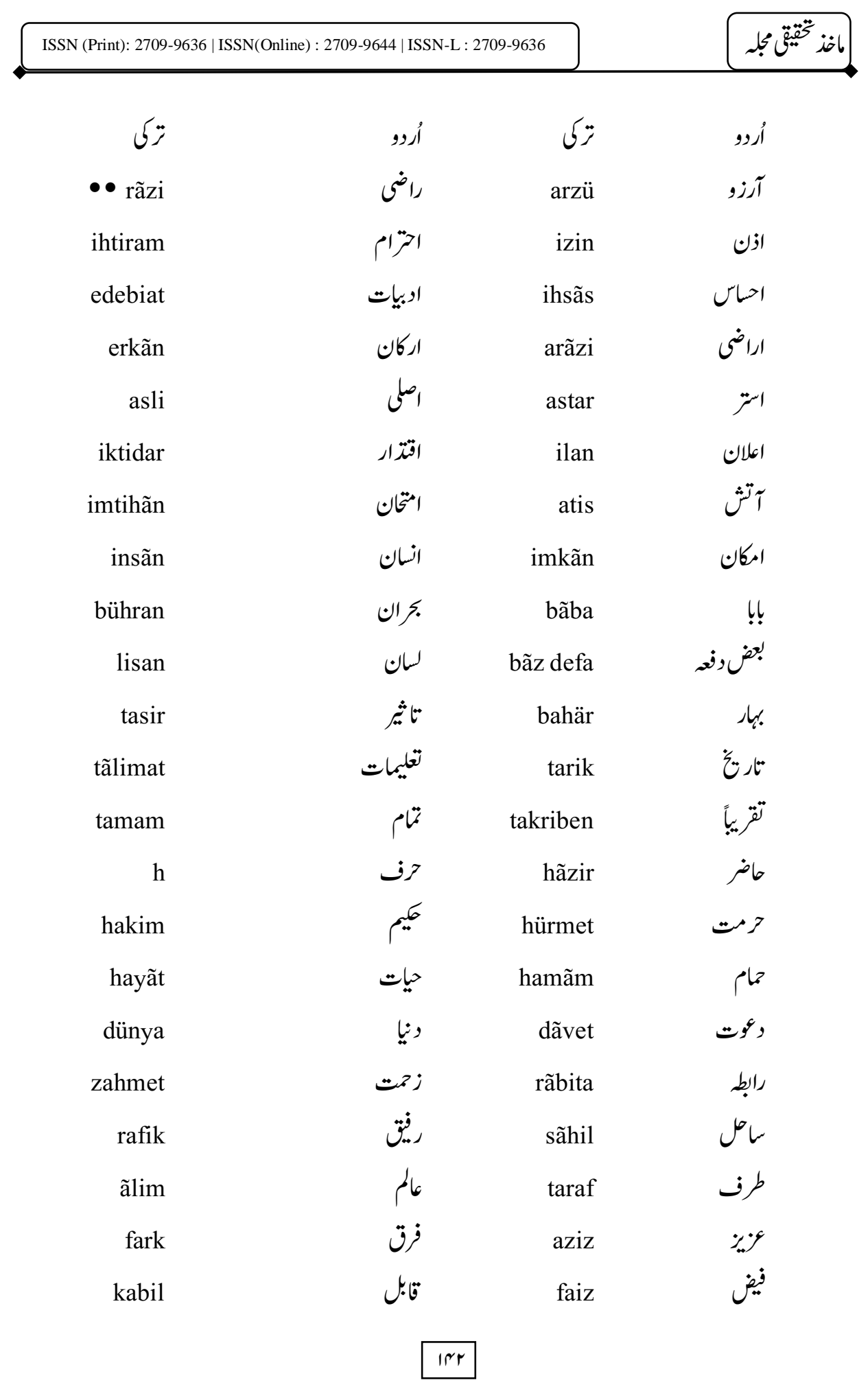




\begin{tabular}{|c|c|c|}
\hline kãfi & 36 & kaza \\
\hline mãlik & كالك & latif \\
\hline matbüa & مطبوء & muhterem \\
\hline müfassal & منصل & meydan \\
\hline nasihat & نضيكت & nadir \\
\hline hafta & بفتخ & $\begin{array}{l}\text { vãkia } \\
{ }_{\left({ }^{(\kappa)}\right)} \text { nazar }\end{array}$ \\
\hline
\end{tabular}

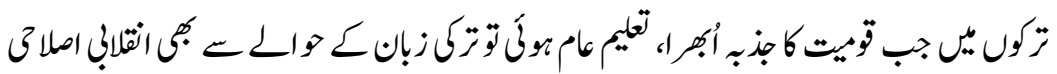

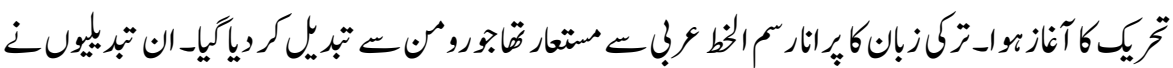

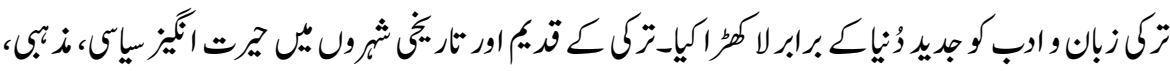

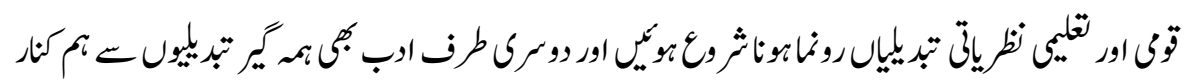

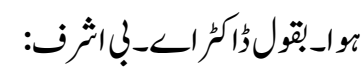

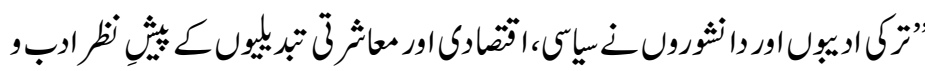

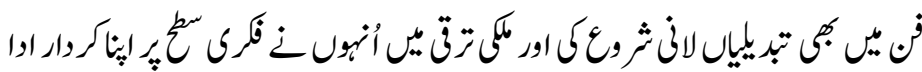

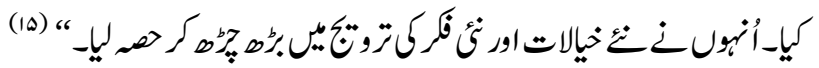

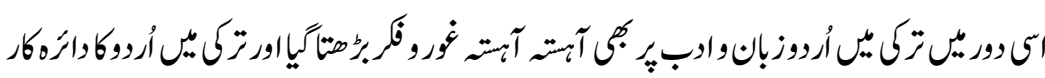

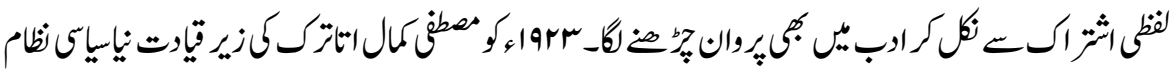

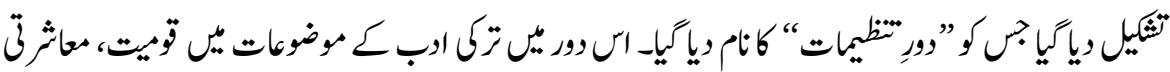

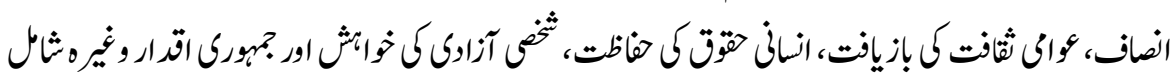

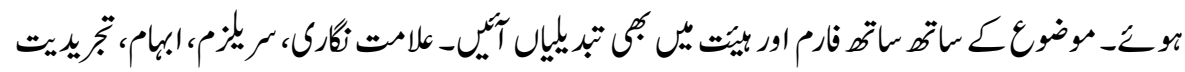

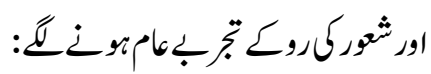

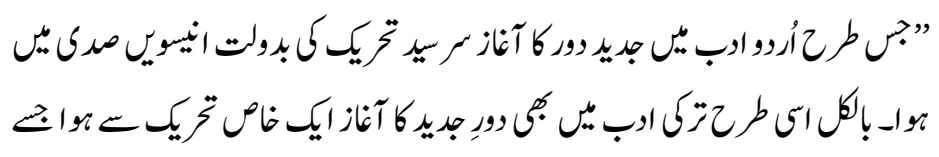




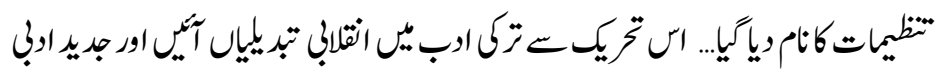

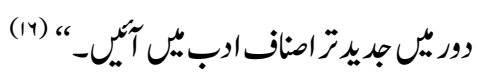

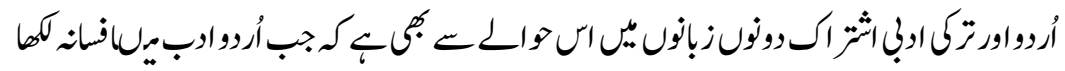

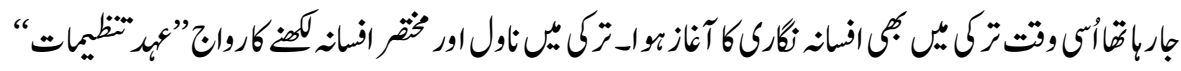

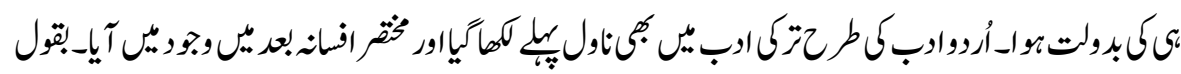

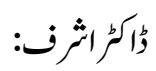

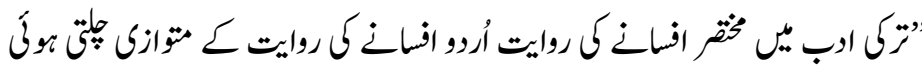

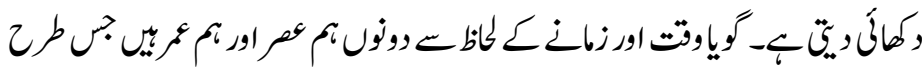

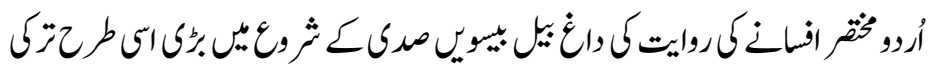

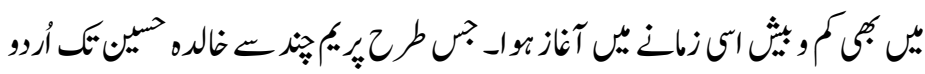

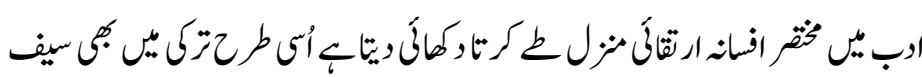

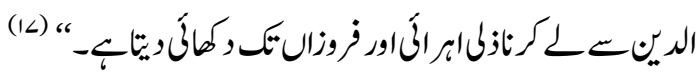

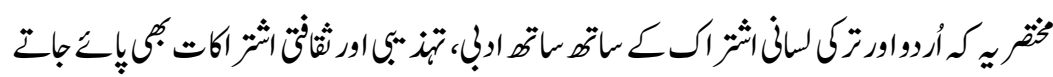

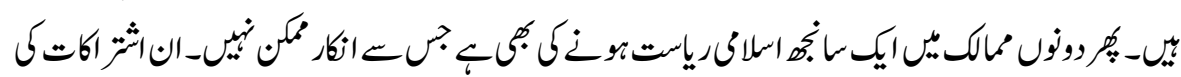

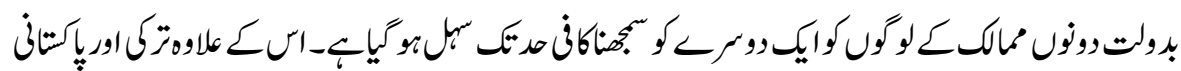

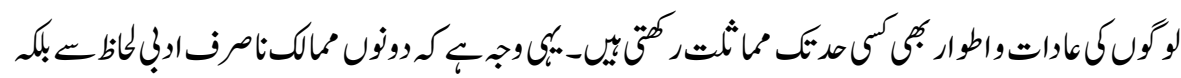

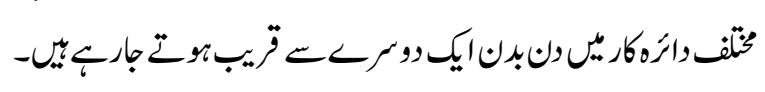

والرجات

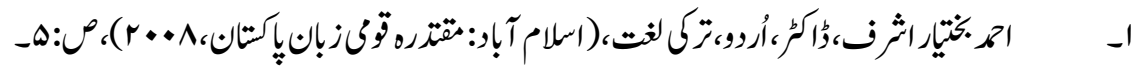

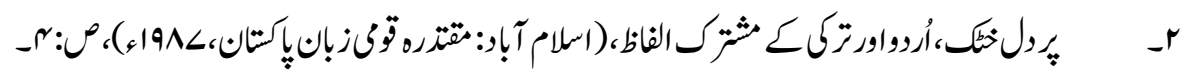

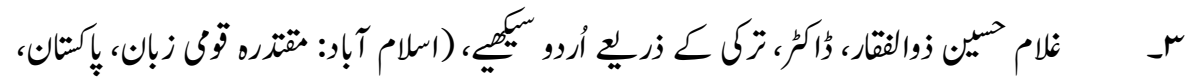
$-r<: 0 \cdot(899 \cdot$ 每 


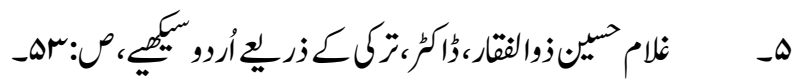

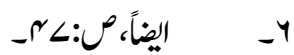

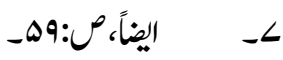

Urdu Research Journal, Issue 11 th,25 July 2017.

Urdu Research Journal, Issue 11 th,25 July 2017.

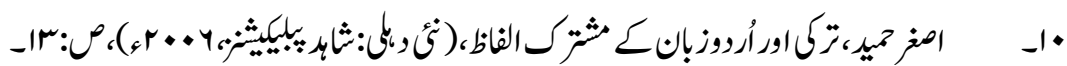

$$
\text { 每 }
$$

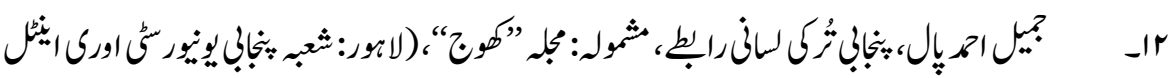

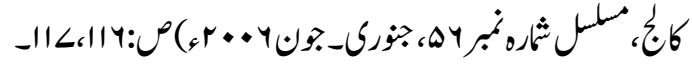

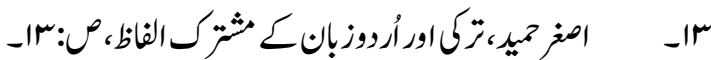

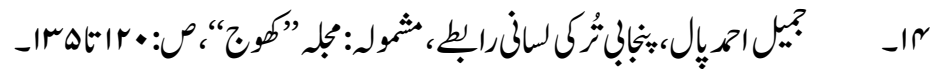

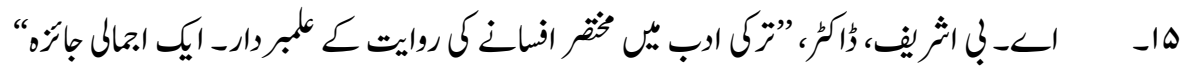

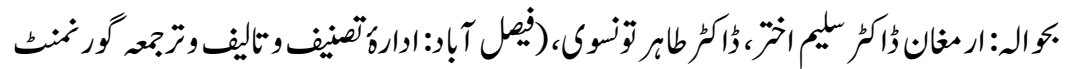

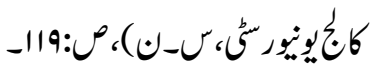

$$
\begin{aligned}
& \text { 14 - إيضاً، ص:119 - }
\end{aligned}
$$

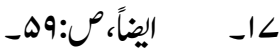

